\title{
Oral Health and Patterns of Dental Visits Among Diabetic Patients in the Eastern Province of Saudi Arabia
}

\author{
Ahmad Mohammed AlTuraiki ${ }^{\prime}$ \\ Hamed Mohammed Jaemal' \\ Ali Ameen Alamer' \\ Ali Ahmed Alghwainem' \\ Thabet Abdulaziz Althabit ${ }^{1}{ }^{\prime}$ \\ Abdulaziz Alamri ${ }^{2}$ \\ Muhammad Ashraf Nazir (D) ${ }^{2}$ \\ 'College of Dentistry Imam Abdulrahman \\ Bin Faisal University, Dammam, Saudi \\ Arabia; ${ }^{2}$ Department of Preventive \\ Dental Sciences, College of Dentistry, \\ Imam Abdulrahman Bin Faisal University, \\ Dammam, Saudi Arabia
}

Purpose: Diabetes is a big public health threat in Saudi Arabia. However, there is limited evidence about dental problems, oral hygiene practices, and dental visits among diabetic patients. The study aimed to evaluate self-perceived oral health status and patterns of dental attendance among diabetic patients in the Eastern province of Saudi Arabia.

Methodology: This cross-sectional study included 486 diabetic patients (mean age $50.14 \pm 13.59$ years) registered with the Saudi Diabetes Society, Saudi Arabia (October-November 2020). The World Health Organization's oral health questionnaire for adults was administered to collect information about oral health status, oral hygiene practices, and dental attendance, in addition to sociodemographic data. Descriptive and analytical statistics were performed.

Results: Most participants (66.3\%) reported having dental pain or discomfort during the last year. Bad breath $(60.50 \%)$, tooth cavities $(55.10 \%)$, difficulty in biting hard food $(55.10 \%)$, tooth sensitivity $(53.30 \%)$, and bleeding gums $(52.90 \%)$ were common oral problems among participants. More than half the sample (55.1\%) reported visiting the dental office during the last year. However, pain or trouble with teeth, gums or mouth was the most common (40.5\%) reason for the last dental visit. Only $7.8 \%$ of participants visited the dentist for a routine dental check-up. Multiple logistic regression analysis showed that Saudi nationality (AOR 2.68, P 0.008), family history of diabetes (AOR 1.88, P 0.022), and having pain during the last year (AOR 4.01, $\mathrm{P}<0.001$ ) were the significant factors associated with the last dental visit due to pain.

Conclusion: This sample of participants demonstrated a high prevalence of dental problems. Few participants performed routine dental visits and most visits were due to pain. Saudis and patients with family history of diabetes and dental pain were more likely to perform pain related dental visits. Multidisciplinary efforts are needed to develop and implement preventive educational and clinical care models for positive oral and general health outcomes.

Keywords: access to oral care, oral health, dental problems, diabetes

\section{Introduction}

Diabetes mellitus is a disorder of insulin either in its production or action and it is associated with body organ damage, dysfunction, or even failure. ${ }^{1}$ There were 366 million diabetic patients globally in 2011, and this number was expected to increase to 552 million by $2030 .^{2}$ According to the World Health Organization (WHO), the prevalence of diabetes mellitus in Saudi Arabia was the second highest in the Middle Eastern region and it was ranked the seventh worldwide. ${ }^{3}$

Dental visits are important for the provision of necessary dental treatment and maintenance of optimal oral health among diabetes patients. ${ }^{4}$ Early management of dental conditions can lead to positive outcomes for natural and functional dentition.
Correspondence: Muhammad Ashraf Nazir Tel +966-543569615

Email manazir@iau.edu.sa 
Most populations do not regularly visit the dentist in developing countries, ${ }^{5}$ whereas about half of the population $(52 \%)$ in the US performs regular dental visits. ${ }^{6}$

Patients without diabetes are more likely to visit a dentist than those with diabetes. ${ }^{7}$ A National Health Survey in the U.S showed that diabetic adults were less likely to visit the dentist in the previous year in comparison with non-diabetic adults (56.8\% diabetic to $64.7 \%$ non-diabetic). ${ }^{8}$ It was also reported that the diabetic adults who visited the dentists were more likely to receive periodontal treatment, tooth extraction, and removable prosthesis. $^{9}$

Evidence showed that more than $86 \%$ of dentists educate diabetic patients about the complications of diabetes on periodontal and oral tissues. ${ }^{10}$ Similarly, a vast majority of physicians and dentists were shown to advise diabetic patients to visit the dental office for a regular dental checkup. ${ }^{11}$ However, most diabetic patients were found to have low awareness about oral problems and lacked the need for preventive dental care. ${ }^{12}$ In fact, lack of knowledge about dental health was one of the reasons for nonadherence to oral hygiene practices in diabetic patients. ${ }^{13}$

Diabetes is one of the biggest public health threats in Saudi Arabia and is strongly associated with negative oral health outcomes. ${ }^{14}$ However, there is limited data about oral health status and dental attendance among diabetic patients in Saudi Arabia. Therefore, it is critical to understand dental problems, oral hygiene behaviors, and patterns of dental visits among diabetic patients. The study aimed to evaluate self-perceived oral health status and dental visits among diabetic patients in the Eastern province of Saudi Arabia.

\section{Materials and Methods}

\section{Study Design and Participants}

This cross-sectional study included adult diabetic patients from the Eastern Province of Saudi Arabia. Diabetic patients (both male and female) were recruited from the Saudi Diabetes Society (October-November 2020). The Society provides education and management of diabetes to the Saudi and non-Saudi populations in the country. Adult diabetic patients from the Eastern province and those who agreed to voluntary participation were eligible to participate in the study. A sample of 547 patients, calculated based on $95 \%$ confidence interval, the precision of estimate (4\%), expected proportion (65\%), and sampling frame $(\geq 20,000)$, was adequate for valid study results.

\section{Measurement of Study Variables}

The World Health Organization's Oral Health Questionnaire for Adults was used to collect information about the oral health status of the participants, oral hygiene practices, dental attendance, and medical history, in addition to sociodemographic information. ${ }^{15}$ The structured questionnaire was divided broadly into three sections. The first section of the questionnaire inquired about gender, age, level of education, medical history, and duration of diabetes. The questions about oral health status were included in the second section of the questionnaire. The participants were asked about tooth cavities, bad breath (malodor), bleeding gums, tooth sensitivity, loosening of teeth/ tooth mobility, dental pain or discomfort, difficulty in biting and chewing food, embarrassed due to the appearance of teeth, and feeling tense because of dental problems. The responses about oral hygiene practices were also sought in this section. The third section included questions about dental visits. The patients provided their responses about the reason for dental visits, types of dental visit, and duration of the last dental visits. The questions about patients' willingness to receive instructions about oral health were included in the last section of the questionnaire. The questionnaire was drafted in English language and discussed among researchers, then it was translated into the Arabic language according to a previously validated Arabic version. ${ }^{16}$ The Arabic version of the questionnaire was reviewed for cultural adaption by an Arab faculty member outside of the study. Arabic questionnaire was administered among study participants. A pilot testing of the Arabic questionnaire was done on 20 diabetic patients in Dammam and AlKhobar to assess their understanding of the questionnaire. However, no changes were indicated in pilot testing and data of these patients were not included in the final analysis.

\section{Study Procedure and Ethics}

The study (EA 202002) was approved by ethics committee at the College of Dentistry, Imam Abdulrahman Bin Faisal University, Dammam, Saudi Arabia. The permission to conduct the study was obtained from the administration of the Saudi Diabetes Society who sent an online questionnaire produced in Google Drive through WhatsApp contacts to its registered diabetic patients in the Eastern province. The participants were introduced to the consent 
form and those willing to participate continued with filling up an online questionnaire. The consent form included aim and objectives of the study including its benefits and the privacy and confidentiality of participants' responses. The study was conducted in accordance with the guidelines of the Helsinki Declaration.

\section{Statistical Analysis}

Data were analyzed using Statistical Package for Social Sciences (IBM SPSS Statistics for Windows, Version 25.0. Armonk, NY: IBM Corp). Means, and standard deviations were calculated for quantitative variables and frequencies and percentages for qualitative variables of the study. Chisquare test was used for bivariate analysis of independent variables (age, gender, education, etc.) with dependent variable (pain related last dental visit). The multiple logistic regression analysis was performed to assess the influence of independent variables on dependent variable. Statistical significance was set at $\mathrm{p}<0.05$.

\section{Results}

The study included data of 486 patients with a mean age of $50.14 \pm 13.59$ years. There were $66.3 \%$ of males and $33.7 \%$ of females in the study. Most participants were Saudis (88.5\%) and educated (91.2\%). The majority of participants (88.5\%) had a family history of diabetes and reported dental pain or discomfort during the past 12 months (66.3\%) Regarding daily oral hygiene behaviors, $61.5 \%$ reported brushing teeth, using mouth wash (21.8\%), and using dental floss $(12.6 \%)$ every day. The study also showed that $86.4 \%$ of participants were willing to receive oral health instructions to improve their oral health (Table 1). The most common oral problems included bad breath $(60.50 \%)$, tooth cavities $(55.10 \%)$, difficulty in biting hard food $(55.10 \%)$, tooth sensitivity (53.30\%), and bleeding gums (52.90\%). On the other hand, difficulty in speech $(15.20 \%)$, oral ulcers $(23.30 \%)$, and tooth mobility $(24.30 \%)$ were the least common problem in the study (Figure 1).

Table 2 displays data of timing and reasons for the last dental visit and reasons for not visiting the dentist. Almost half the sample $(55.1 \%)$ reported visiting dental office during the last year. However, pain or trouble with teeth, gums, or mouth was the most common (40.5\%) reason for the last dental visit. Only $7.8 \%$ of the participants visited the dentist for a routine dental check-up. Difficulty in getting an appointment (29.8\%) and non-affordability of dental treatment $(29.2 \%)$ were the most common reasons for not visiting the dentist.
Table I Demographic and Oral Health Information of Study Participants

\begin{tabular}{|c|c|}
\hline Variables & $\begin{array}{l}\text { Frequency }(\%) \mathrm{N}= \\
486\end{array}$ \\
\hline \multicolumn{2}{|l|}{ Gender: } \\
\hline Male & $322(66.3)$ \\
\hline Female & $164(33.7)$ \\
\hline \multicolumn{2}{|l|}{ Age } \\
\hline Adults (22-64 years) & $415(85.4)$ \\
\hline Older adults ( $\geq 65$ years) & $71(14.6)$ \\
\hline \multicolumn{2}{|l|}{ Nationality: } \\
\hline Saudi. & $430(88.5)$ \\
\hline Non-Saudi & $56(11.5)$ \\
\hline \multicolumn{2}{|l|}{ Level of education: } \\
\hline No education. & $43(8.8)$ \\
\hline School education. & $258(53.1)$ \\
\hline University or higher level. & $185(38.1)$ \\
\hline \multicolumn{2}{|l|}{ Level of Income } \\
\hline Low: less than $5000 \mathrm{SAR} /$ month & $213(43.8)$ \\
\hline Middle: $500 \mathrm{I}-20,000 \mathrm{SAR} /$ month & $248(51.0)$ \\
\hline High: $20,000 \mathrm{SAR} / \mathrm{month}$ and above & $25(5.1)$ \\
\hline \multicolumn{2}{|l|}{ Family history of Diabetes: } \\
\hline Yes & $430(88.5)$ \\
\hline No & $56(11.5)$ \\
\hline \multicolumn{2}{|l|}{ Diabetes duration } \\
\hline $0-10$ years & $379(78.0)$ \\
\hline More than 10 years & $107(22.0)$ \\
\hline \multicolumn{2}{|l|}{$\begin{array}{l}\text { Dental pain or discomfort during the past } 12 \\
\text { months }\end{array}$} \\
\hline Yes. & $322(66.3)$ \\
\hline No. & 121 (24.9) \\
\hline I do not remember/know & $43(8.8)$ \\
\hline \multicolumn{2}{|l|}{ Oral hygiene behaviors } \\
\hline \multicolumn{2}{|l|}{ Daily tooth brushing } \\
\hline Yes & $299(61.5)$ \\
\hline No & $187(38.5)$ \\
\hline \multicolumn{2}{|l|}{ Daily use of mouth wash } \\
\hline Yes & $106(21.8)$ \\
\hline No & $380(78.2)$ \\
\hline \multicolumn{2}{|l|}{ Daily use of dental floss } \\
\hline Yes & $61(12.6)$ \\
\hline No & $425(87.4)$ \\
\hline \multicolumn{2}{|l|}{ Willingness to receive oral health instructions } \\
\hline Yes & $420(86.4)$ \\
\hline No & $66(13.6)$ \\
\hline
\end{tabular}

Results of bivariate analysis and multiple logistic regression analysis are shown in Table 3. In bivariate analysis, Saudi participants (OR 3.12, P 0.001), those with a family history of diabetes (OR 2.12, P0.003), and those who had pain during the last year (OR 4.12, 


\section{Distribution of dental problems}

Difficulty with speech

Oral ulcers

$15.20 \%$

Tooth mobility

Felt tense because of problem with teeth or gums

Difficulty in chewing food

Difficulty in chewing food
Felt embarasshed due to appearance of teeth

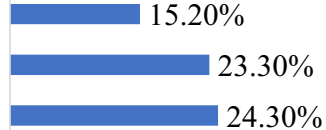

Bleeding gums

Tooth Sensitivity

Difficulty in biting hard food

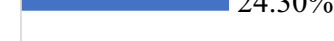

$43.60 \%$

$44.20 \%$

$47.30 \%$

$52.90 \%$

$53.30 \%$

$55.10 \%$

Tooth Cavities

Bad Breath

Figure I Distribution of participants' responses about the self-perceived oral problems.

$\mathrm{P}<0.001)$ were significantly more likely to perform pain related dental visit. Saudi nationality (AOR 2.68, P 0.008), family history of diabetes (AOR 1.88, P 0.022), and having pain during the last year (AOR 4.01, $\mathrm{P}<0.001$ ) were the significant factors associated with the last dental visit due to pain after controlling for other factors.

Figure 2 provides information about sources of oral health instructions among study participants. Dental office (50\%) was the most source of oral health instructions, followed by social media (30.7\%), and family and friends (28\%).

\section{Discussion}

Saudi and non-Saudi diabetic patients in the present study demonstrated a high prevalence of dental pain, bad breath, tooth cavities, difficulty in biting hard food, tooth sensitivity, and bleeding gums. The literature also indicates the

Table 2 Dental Attendance Patterns Among Study Participants

\begin{tabular}{|c|c|}
\hline Variables & Frequency (\%) \\
\hline \multicolumn{2}{|l|}{ How long is it since you last saw a dentist? } \\
\hline$\leq \mathrm{I}$ year. & $268(55.1)$ \\
\hline$>$ I year but $<2$ years. & $76(15.6)$ \\
\hline$\geq 2$ years but $<5$ years. & $91(18.7)$ \\
\hline$\geq 5$ years. & $51(10.5)$ \\
\hline \multicolumn{2}{|l|}{ What was the reason of your last visit to the dentist? } \\
\hline Consultation/advice. & $52(10.7)$ \\
\hline Pain or trouble with teeth, gums or mouth. & $197(40.5)$ \\
\hline Treatment/ follow-up treatment. & $127(26.1)$ \\
\hline Routine check-up/treatment. & $38(7.8)$ \\
\hline Do not know/do not remember. & $72(14.8)$ \\
\hline \multicolumn{2}{|l|}{ Reasons for not visiting the dentist } \\
\hline I do not have dental insurance. & III (22.8) \\
\hline I cannot afford to have dental treatment. & $142(29.2)$ \\
\hline I do not have time to visit the dentist. & $72(14.8)$ \\
\hline I have dental anxiety/ I am afraid of dental treatment. & $73(15.0)$ \\
\hline I must travel a long distance to receive dental treatment. & $2(0.4)$ \\
\hline It is difficult to get a dentist's appointment. & $145(29.8)$ \\
\hline I am not satisfied with the quality of oral care services. & $29(6.0)$ \\
\hline I do not think oral care is important for good health. & $26(5.3)$ \\
\hline
\end{tabular}


Table 3 Association of Factors with the Last Dental Visit Due to Pain Among Study Participants

\begin{tabular}{|c|c|c|c|c|}
\hline Variables & Unadjusted Odds Ratio $(95 \% \mathrm{Cl})$ & P-value & Adjusted Odds Ratio (95\% Cl) & P-value \\
\hline $\begin{array}{l}\text { Gender: } \\
\text { Male } \\
\text { Female }\end{array}$ & $\mathrm{I} .10(0.75, \mathrm{I} .6 \mathrm{I})$ & 0.628 & $\mathrm{I} .32(0.86,2.0 \mathrm{I})$ & 0.201 \\
\hline $\begin{array}{l}\text { Age } \\
\text { Adults } \\
\text { Older adults }\end{array}$ & I.2। $(0.72,2.04)$ & 0.467 & $1.03(0.58,1.82)$ & 0.924 \\
\hline $\begin{array}{l}\text { Nationality: } \\
\text { Saudi. } \\
\text { Non-Saudi }\end{array}$ & $3.12(1.57,6.20)$ & 0.001 & $2.68(1.29,5.55)$ & 0.008 \\
\hline $\begin{array}{l}\text { Level of education } \\
\text { No/school education. } \\
\text { University or higher level. }\end{array}$ & $0.67(0.46,0.97)$ & 0.036 & $0.7 \mathrm{I}(0.46, \mathrm{I} .10)$ & 0.125 \\
\hline $\begin{array}{l}\text { Level of income } \\
\text { Low income } \\
\text { Middle/high income }\end{array}$ & $0.86(0.60,1.24)$ & 0.419 & $0.99(0.65,1.50)$ & 0.948 \\
\hline $\begin{array}{l}\text { Family history of Diabetes } \\
\text { Yes } \\
\text { No }\end{array}$ & $2.12(1.28,3.49)$ & 0.003 & I.88 (I.I0, 3.22) & 0.022 \\
\hline $\begin{array}{l}\text { Diabetes duration } \\
0-10 \text { years } \\
\text { More than } 10 \text { years }\end{array}$ & $0.92(0.60,1.43)$ & 0.717 & I.28 $(0.79,2.05)$ & 0.313 \\
\hline $\begin{array}{l}\text { Pain or discomfort during the past } 12 \text { months } \\
\text { Yes. } \\
\text { No. }\end{array}$ & $4.12(2.65,6.40)$ & $<0.001$ & $4.23(2.67,6.67)$ & $<0.001$ \\
\hline
\end{tabular}

high occurrence of pain, periodontal diseases, bad breath/ halitosis, tooth decay, delay wound healing, and other oral complications among diabetic patients. ${ }^{14}$ It is known that hyperglycemia in diabetic patients is associated with the development of periodontal and other oral diseases. ${ }^{14}$ Hyperglycemia disrupts salivary composition and functions and increases cytokines and other inflammatory mediators in saliva and gingival crevicular fluid leading to exacerbation of inflammation and deterioration of periodontal and oral tissues. ${ }^{14,17}$ In addition, reduced salivary flow diminishes cleansing and buffer capacity of saliva and enhances plaque accumulation thus compromising oral health of diabetic patients. ${ }^{18}$ Bad breath was common in our sample which can be related to the production of volatile sulfur compounds from odoriferous microorganisms. ${ }^{19}$ These oral complications in diabetic patients are known to lower their quality of life. ${ }^{20}$

Diabetes is associated with multiple health risks, however, regular dental care visits are known for positive oral health outcomes for diabetic adults. ${ }^{8}$ A study by Lopez et al found that regular dental visits for diabetic patients resulted in a significant improvement in probing depth, bleeding on probing, and sites with plaque after 3 months. ${ }^{21}$ Despite optimal oral health benefits of regular dental visits, a very small proportion of diabetic patients in our sample performed routine dental check-ups. Similar patterns of fewer regular dental visits were reported in previous studies of diabetic adults in Saudi Arabia. ${ }^{22-24}$ More than $90 \%$ of diabetic patients who visited primary health care centers in Riyadh did not get their routine dental check-up every six months. ${ }^{22}$ A study by $\mathrm{Al}$ Amassi and Al Dakheel found only 15.1\% of diabetic patients regularly visiting the dentist in Saudi Arabia. ${ }^{23}$ Similarly, a low prevalence of dental visits in the last year (13\%) among diabetic patients in Riyadh was reported by Bahammam. ${ }^{24}$ Likewise, only $14 \%$ of diabetic patients in the United Arab Emirates were found to visit a dentist for a regular dental check-up. ${ }^{25}$ This consistent finding of 


\section{Sources of oral health instructions}

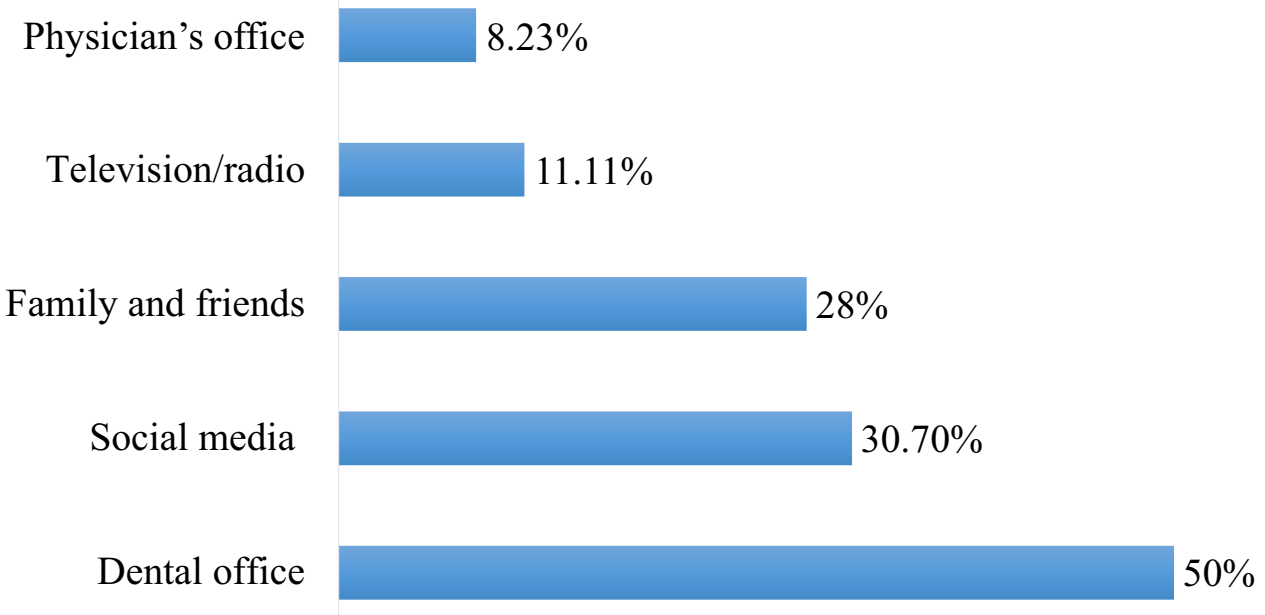

Figure 2 Distribution of participants' responses about sources of oral health instructions.

fewer regular dental visits among diabetic patients was also reported in a systematic review of 28 studies from 14 countries. ${ }^{17}$ Recently, the researchers also observed lower odds of preventive dental visits among diabetic patients than those without diabetes in the U.S. ${ }^{26}$

Dental pain was the most frequent reason for dental visits in our sample. Diabetic patients demonstrate a high prevalence of oral diseases resulting in painful sensation in the oral cavity which may account for an increased likelihood of pain related dental visits in our sample. ${ }^{14,27}$ The present study also showed that Saudi nationals were more likely to perform pain related dental visits. This pattern of increased dental visits due to pain in Saudi Arabia is also supported by the results of a previous study where most adult patients performed pain related dental visits in the Eastern region. ${ }^{28}$ Family history of diabetes is a strong risk factor associated with the development of diabetes. ${ }^{29}$ Therefore, these subjects are more likely to have increased distribution of oral diseases and subsequently dental pain. This may explain why participants with a family history of diabetes were about twice (AOR 1.88) more likely to perform pain related dental visits than those without a family history in the present study.

Difficulty in getting a dental appointment was the most common reason for not performing dental visits in the present study. These findings corroborate with the results of a study by Almutlaqah et al who reported difficulty in getting an appointment as one of the main barriers to dental care in Saudi Arabia. ${ }^{30}$ The affordability of dental treatment was the second most common reason for not visiting the dentist among our sample of diabetic patients. A study by Moore et al indicated that the majority of diabetic patients in the US avoided visiting a dental office because of the cost of dental treatment. ${ }^{12}$ Moreover, a systemic review cited the cost of dental treatment as the main barrier to dental care in diabetic patients. ${ }^{17}$ The lack of insurance was the third most important reason for not visiting the dentist in the present study. There is a significant association between not having health insurance and avoiding the dental visit. ${ }^{31}$

Health care providers can play an important role in improving oral health behaviors of diabetic patients by providing oral health education. ${ }^{17}$ In the present study, the dental office was the main source of oral health education. This is contrary to a study of diabetic patients by $\mathrm{Al}$ Amassi and $\mathrm{Al}$ Dakheel who reported that social media was the main source of oral health information followed by dentists and dental hygienists. ${ }^{23}$ Almaiman et al showed that $67.7 \%$ of social media users seek online oral health related information in Saudi Arabia. ${ }^{32}$ In the present study, $30.70 \%$ of patients indicated social media as an important source of oral health education. Given the important and emerging role of social media, it is suggested that high-quality evidence-based oral health information should be provided through social media outlets to raise awareness about the importance of oral health including access to oral care services.

The present study provided valuable data that may be used to reduce oral health disparity among diabetic patients in Saudi Arabia. However, there are certain limitations to the study. 
Adult diabetic patients from the Eastern province of Saudi Arabia participated in the study, therefore study results should not be generalized to diabetic patients from all over the country. Moreover, the use of a non-probability convenience sample of diabetic patients may lead to bias in the study. A standardized World Health Organization's oral health questionnaire was used in the study to obtain valid and reliable results. However, self-reported data are limited because of under and over reporting of responses. More accurate information about oral problems and dental visits can be obtained by performing clinical oral examination and review of dental records, respectively. However, there were challenges of oral screening of diabetic patients and data collection from clinics due to infection control protocols during the COVID-19 pandemic. Future studies should investigate clinically diagnosed oral problems and associated factors in a large sample of diabetic patients from all regions of Saudi Arabia.

\section{Conclusion}

This study showed a high prevalence of dental pain or discomfort during the past 12 months among diabetic patients. Most participants reported bad breath, tooth cavities, difficulty in biting hard food, tooth sensitivity, and bleeding gums. Pain or trouble with teeth, gums, or mouth was the most common reason for the last dental visit. There was a very low prevalence of routine dental checkups among participants. Significant factors associated with the last dental visit due to pain included Saudi nationality, family history of diabetes, and pain during the past year. The study results underscore the need for public health measures to promote routine dental care and reduce the prevalence of oral problems among diabetic patients.

\section{Acknowledgments}

The authors grateful to the Saudi Diabetes Society for their great help in data collection.

\section{Disclosure}

The authors report no conflicts of interest in this work.

\section{References}

1. Alam U, Asghar O, Azmi S, Malik RA. General aspects of diabetes mellitus. Handb Clin Neurol. 2014;126:211-222.

2. Whiting DR, Guariguata L, Weil C, Shaw J. IDF diabetes atlas: global estimates of the prevalence of diabetes for 2011 and 2030. Diabetes Res Clin Pract. 2011;94(3):311-321. doi:10.1016/j.diabres.20 11.10.029

3. World Health Organization. Global Report on Diabetes. World Health Organization; 2016.
4. Syrjala AM, Kneckt MC, Knuuttila ML. Dental self-efficacy as a determinant to oral health behaviour, oral hygiene and $\mathrm{HbA1c}$ level among diabetic patients. J Clin Periodontol. 1999;26 (9):616-621. doi:10.1034/j.1600-051X.1999.260909.x

5. Nagarajappa R, Sahare P, Shukla N, Tuteja J, Bhalla A. Knowledge, attitude and practices regarding oral health among out patients at Rama dental college, Kanpur. Rama Univ J Dent Sci. 2015;2(1):8-14.

6. American Dental Association. The Adult's Oral Health \& Well-Being Survey. Available from: https:/www.ada.org/en/science-research/healthpolicy-institute/oral-health-and-well-being. Accessed September 13, 2019.

7. Lalla E, Park DB, Papapanou PN, Lamster IB. Oral disease burden in northern Manhattan patients with diabetes mellitus. Am J Public Health. 2008;98(9 Suppl):S91-S94. doi:10.2105/AJPH.98.Supplement_1.S91

8. Macek MD, Tomar SL. Dental care visits among dentate adults with diabetes and periodontitis. J Public Health Dent. 2009;69 (4):284-289. doi:10.1111/j.1752-7325.2009.00136.x

9. Chaudhari M, Hubbard R, Reid RJ, et al. Evaluating components of dental care utilization among adults with diabetes and matched controls via hurdle models. BMC Oral Health. 2012;12:20. doi:10.1186/ 1472-6831-12-20

10. Esmeili T, Ellison J, Walsh MM. Dentists' attitudes and practices related to diabetes in the dental setting. J Public Health Dent. 2010;70(2):108-114. doi:10.1111/j.1752-7325.2009.00150.x

11. Bahammam MA. Awareness and practice about the association between periodontal diseases and diabetes mellitus: a cross-sectional study in Western Saudi Arabia among health care providers. J Multidiscip Healthc. 2018;11:511-517. doi:10.2147/ JMDH.S177143

12. Moore PA, Orchard T, Guggenheimer J, Weyant RJ. Diabetes and oral health promotion: a survey of disease prevention behaviors. $J$ Am Dent Assoc. 2000;131(9):1333-1341. doi:10.14219/jada.archive.2000.0388

13. Ismaeil FM, Ali N. Diabetic patients knowledge, attitude and practice toward oral health. JEP. 2013;4(20):19-25.

14. Nazir MA, AlGhamdi L, AlKadi M, AlBeajan N, AlRashoudi L, AlHussan M. The burden of diabetes, its oral complications and their prevention and management. Open Access Maced J Med Sci. 2018;6(8):1545-1553. doi:10.3889/oamjms.2018.294

15. World Health Organization. Oral Health Surveys: Basic Methods. World Health Organization; 2013.

16. Khoshnevisan MH, Albujeer AN, Attaran N, Almahafdha A, Taher A. WHO's oral health assessment questionnaire for adult: psychometric properties of the Arabic version. J Contemp Med Sci. 2016;2(8):116-118. doi:10.22317/jcms.2016122

17. Poudel P, Griffiths R, Wong VW, et al. Oral health knowledge, attitudes and care practices of people with diabetes: a systematic review. BMC Public Health. 2018;18(1):577. doi:10.1186/s12889018-5485-7

18. Seethalakshmi C, Reddy RC, Asifa N, Prabhu S. Correlation of salivary $\mathrm{pH}$, incidence of dental caries and periodontal status in diabetes mellitus patients: a cross-sectional study. J Clin Diagn Res. 2016;10(3):Zc12-4. doi:10.7860/JCDR/2016/16310.7351

19. Kamaraj DR, Bhushan KS, Laxman VK, Mathew J. Detection of odoriferous subgingival and tongue microbiota in diabetic and nondiabetic patients with oral malodor using polymerase chain reaction. Indian $J$ Dent Res. 2011;22(2):260-265. doi:10.4103/0970-9290.84301

20. Cervino G, Terranova A, Briguglio F, et al. Diabetes: oral health related quality of life and oral alterations. Biomed Res Int. 2019;2019:5907195. doi:10.1155/2019/5907195

21. Lopez NJ, Quintero A, Casanova PA, Martinez B. Routine prophylaxes every 3 months improves chronic periodontitis status in type 2 diabetes. J Periodontol. 2014;85(7):e232-40. doi:10.1902/jop.2013.130400

22. Aljaber A, Al-Surimi K. Promoting oral health practice among patients with diabetes attending primary health care clinics. $B M J$ Qual Improv Rep. 2015;4(1):u209172.w3737. doi:10.1136/bmjquality.u209172.w3737 
23. Al Amassi BY, Al Dakheel RS. Oral hygiene practice of adult diabetic patients and their awareness about oral health problems related to diabetes. J Dent Oral Hyg. 2017;9(2):8-14. doi:10.5897/ JDOH2017.0219

24. Bahammam MA. Periodontal health and diabetes awareness among Saudi diabetes patients. Patient Prefer Adherence. 2015;9:225-233. doi:10.2147/PPA.S79543

25. Eldarrat AH. Diabetic patients: their knowledge and perception of oral health. Libyan J Med. 2011;6(1):5691. doi:10.3402/ljm. v6i0.5691

26. Zhang Y, Leveille SG, Shi L, Camhi SM. Disparities in preventive oral health care and periodontal health among adults with diabetes. Prev Chronic Dis. 2021;18:E47. doi:10.5888/pcd18.200594

27. Ahmad R, Haque M. Oral health messiers: diabetes mellitus relevance. Diabetes Metabol Syndr Obes. 2021;14:3001. doi:10.2147/DMSO.S318972

28. Nazir MA. Factors associated with dental pain related to last dental visit among adult patients. Dent Med Probl. 2018;55(1):63-68. doi:10.17219/dmp/83039
29. Scott RA, Langenberg C, Sharp SJ, et al. The link between family history and risk of type 2 diabetes is not explained by anthropometric, lifestyle or genetic risk factors: the EPIC-InterAct study. Diabetologia. 2013;56(1):60-69.

30. Almutlaqah MA, Baseer MA, Ingle NA, Assery MK, Al Khadhari MA. Factors affecting access to oral health care among adults in Abha City, Saudi Arabia. J Int Soc Prev Commun Dent. 2018;8(5):431-438. doi:10.4103/jispcd.JISPCD_205_18

31. Centers for Disease Control and Prevention (CDC). Dental visits among dentate adults with diabetes-United States, 1999 and 2004. MMWR. 2005;54(46):1181-1183.

32. Almaiman S, Bahkali S, Alabdulatif N, et al. Promoting oral health using social media platforms: seeking Arabic Online Oral Health Related Information (OHRI). Stud Health Technol Inform. 2016;226:283-286.

\section{Publish your work in this journal}

Clinical, Cosmetic and Investigational Dentistry is an international, peer-reviewed, open access, online journal focusing on the latest clinical and experimental research in dentistry with specific emphasis on cosmetic interventions. Innovative developments in dental materials, techniques and devices that improve outcomes and patient satisfaction and preference will be highlighted. The manuscript management system is completely online and includes a very quick and fair peer-review system, which is all easy to use. Visit http://www.dovepress.com/testimonials.php to read real quotes from published authors. 